\title{
Twenty-five years since the Shearman Report: How far have we come? Are we there yet?
}

\section{ABSTRACT \\ Background}

In 1989, the first major state-wide report into maternity services, known as the Shearman report after its author, was released in New South Wales, the most populous state in Australia.

\begin{abstract}
Aim
This paper reflects upon the report, and tracks the progress of five of its key recommendations. The recommendations are still some of the major issues facing maternity services across the country. These are: community-based maternity care; rural maternity services; hospital visiting rights for privately-practising midwives; obstetric intervention; and midwifery continuity of maternity care.
\end{abstract}

\section{Findings}

In some ways, much has changed in 25 years including the terminology used in the report, the importance of midwifery continuity of care and the woman-centred nature of many services. However, in other ways, there is still a long way to go to address these major issues. Despite more than a quarter of a century, many recommendations have not been fulfilled, especially access to care in rural areas, rates of obstetric intervention, and the issue of visiting rights for privately-practising midwives which has gone backwards.

\section{Conclusion}

A continued and renewed effort is needed to ensure that the forward thinking recommendations of the Shearman Report are ultimately realised for all women and their families.

Keywords: The Shearman Report; Maternity reports; New South Wales; Australia 


\section{INTRODUCTION}

Over the past 25 years, Australian maternity services have been influenced by a number of state and national reviews and reports (see Figure 1). The first of these was released in New South Wales (NSW), the most populous state, in $1989 .{ }^{1}$ The report was a result of a Ministerial Task Force established in 1987 to address issues in maternity care in NSW. In the preceding years, concern had been mounting over the increasing medicalisation of childbirth and the limited rights and capacity of women to participate in decision-making regarding their pregnancy and birth care. Childbirth had become increasingly medicalised and pathological, women had little or no say in their care and there were few specific services for women from minority or disadvantaged groups. During the 1960 s and 70 s it was quite normal that medical doctors and the health system determined women's childbearing needs. ${ }^{2}$ However, pressure from the vocal women's movement that challenged medical dominance and views of safety, and the professionalisation of midwives, meant greater autonomy for childbearing women. ${ }^{3,4}$ It was in this highly charged environment in mid 1980 s that it was evident that change was needed - hence a number of reviews occurred, $\underline{\text { the first of these was the result of the Ministerial Task Force. }}$

The Taskforce, chaired by obstetrician Dr Rodney Shearman, began a dialogue between maternity care providers, health service planners and the women of NSW. This involved consumer satisfaction surveys, over 2500 people attending 30 public and 28 provider forums, visits to 30 maternity units, and nearly 300 written submissions from interested parties. In 1989, the Final Report of the Ministerial Task Force on Obstetric Service in NSW was released, commonly known as the Shearman Report. ${ }^{1}$

The Shearman Report advocated several important principles that lay the foundation of the report. These were that women had a right to equitable access to quality maternity care, should participate in decision-making about their care and that of their babies, and that they should be cared for by professionals who work in collaboration with each other. A 
redistribution of hospital beds was proposed to accommodate populations in the south and west of Sydney, and more neonatal services and community support were suggested. The need for equity of antenatal care services - especially for disadvantaged populations, and those that did not speak English as a first language, were highlighted. The professional skills of obstetricians and midwives were examined, and systems of continuing professional development suggested.

Most importantly, this report recommended that birth be approached as a natural event which was somewhat at odds with the prevailing environment of pathology. This meant the need to provide more home-like rooms and units, increasing numbers of birth centres, and a careful monitoring of medical intervention rates which were on the rise. The report also suggested that postnatal care of well women could be moved out of the hospital setting and community midwifery care be expanded.

Much has happened in more than 25 years since the release of the Shearman Report. Some aspects of maternity care have changed considerably while other issues are remarkably similar and the medical intervention rates have escalated beyond what anyone probably foreshadowed at the time. In the intervening years, more than 16 major state or national reports have been published into maternity services in Australia many of which have recommended similar issues (see Figure 1).

The aim of this Commentary is to reflect upon the Shearman Report and specifically track the progress of five key recommendations in NSW that were made in 1989 through to 2015. The recommendations pertain to some of the major issues that have been addressed through many of the other reports, that is: community-based maternity care; rural maternity services; hospital visiting rights for privately-practising midwives; obstetric intervention; and midwifery continuity of maternity care. The final part of the Commentary proffers some views as to the lack of movement in some critical areas and poses some challenges for policy makers, professional organisations, clinician and consumers of how to move forward. 
PUT FIGURE 1 HERE

\section{Community-based maternity care}

Recommendation 3.4: that the extension of existing public hospital antenatal clinics to the community be encouraged and the problems affecting access to public hospital clinics (eg transport) be addressed (p. 162).

The Shearman Report recommended the expansion of community-based maternity care through the utilisation of outreach services in community health centres, and child and family health centres (previously called 'early childhood centres'). It was recognised that women in rural areas in particular had issues with transport and access to services, so to help ease this problem it was recommended that midwives working in the community could provide antenatal care, and that health services investigate after-hours antenatal services.

Similar recommendations regarding the expansion of outreach clinics and postnatal early discharge programs were apparent in subsequent state and national reports. For example, the NSW Framework for Maternity Services $(2000)^{5}$ (the 'NSW Framework report') and the National Maternity Services Plan $(2010)^{6}$ both recommended improved access to community-based antenatal and postnatal services.

There are currently no 2015 data on how many NSW hospitals have community-based antenatal clinics or other services that specifically address access. However, 11 years after the Shearman Report, the NSW Framework Report ${ }^{5}$ showed that the 72 public hospitals in NSW (which was the majority providing maternity care at the time) had an early discharge and/or community midwifery program that cared for postnatal women and babies, although it was unclear as to how many midwives working in the community provided antenatal care in addition to postnatal care. 
Caring for pregnant and postnatal women in community settings and in their homes supports ease of women's access to clinics within their community, promotes family involvement, and negates the need for well women to travel to or stay within a hospital. In particular, for disadvantaged groups, and some Aboriginal communities, having care in the community can increase participation and attendance, which can lead to better maternal and neonatal outcomes. Community-based maternity care comprises outreach antenatal clinics, homebirth services and postnatal community midwifery programs. Providing continuity of care in women's homes in collaboration with GP and/or specialist support, is highly rated and associated with positive outcomes. ${ }^{7}$

The Shearman report not only called for more community-based care, but recommended 'that consideration be given to providing a more home-like atmosphere in antenatal wards' (recommendation 3.14, p. 172) for the overall comfort of women. It also called for the better organisation of antenatal care. This meant instigating appointment systems (to respond to unacceptable waiting times), improving public transport to clinics, and provision of on-site supervised child care facilities during clinic times. The report also stated that investigation be made regarding the provision of financial incentives (to attend the clinic) for disadvantaged women.

Despite these strategies to address access, it would seem that apart from appointment systems being implemented in most clinics very few (if any) institutions provide child care facilities for women attending clinics, and no government has instigated financial incentives for women to attend clinics.

\section{Rural maternity services}

Recommendation 2.1: The viability of small country maternity units should be determined by: the availability of appropriately qualified medical and midwifery staff to manage normal confinements and cope with unexpected complications pending transfer; the degree of geographic isolation; and continued demand reflecting trends in population growth or 
decline. Number of births should not be the sole criterion for determining unit viability unless contiguous units are each underutilised in which case amalgamation may need to be considered (p. 97).

There were many issues in the Shearman Report in relation to women living in rural areas and the lack of maternity services (diagnostic, antenatal, birth and postnatal) in these areas. Issues included the transport and financial difficulties women faced when needing to travel long distances to obstetric services; and a lack of Aboriginal midwives and Aboriginal health workers (AHW). It was thought that with improvements in antenatal care, rural and remote women would be able to stay within their communities longer and they would transfer to hospital prior to birth at a later gestation.

Prior to the Shearman Report, 35 small rural maternity units in NSW had closed due to a 1983 report stating that units were 'non-viable' with fewer than 80 births per annum. ${ }^{8}$ The Report found that women felt the services provided a personal care and the units were often the cornerstone of the community; it was noted that their loss often preceded the erosion of other medical and hospital services.

The other issues for women from rural and remote communities were about finding suitable accommodation for themselves and their families when transferred to hospital to await birth. Shearman suggested that this be provided not only for the women, but their families too. Objectives in the NSW Framework report ${ }^{5}$ also repeated the need to increase equity of maternity service access for women from rural and remote areas and suggested exploring options for developing support services to help families, such as childcare, transport and accommodation services.

Since 1996, NSW Health Department funding initiatives have enabled the development of several rural health workforce strategies. These are: support for expansion of medical specialty and refresher training positions in rural areas, locum services to provide rural 
obstetricians with relief, and recruitment of rural GPs with obstetric training. Moves to help midwifery and nursing disciplines have also been made with two rural/remote professorial chairs created, a rural/remote nursing scholarship fund, a maternity emergencies survival package for registered nurses, midwifery refresher courses and materials to enhance recruitment of nurses and midwives to these areas. ${ }^{5}$ However, despite the strong rural focus of the National Maternity Services Plan ${ }^{6}$ women in rural and remote areas still have poor access to maternity services. Some of the major reasons for this is the lack of specialised medical and midwifery staff, and the closure of over half of Australia's rural maternity units over the last 15 years due to perceptions of safety risks, cost and workforce shortages. ${ }^{9}$

\section{Aboriginal and Torres Strait Islander women and their communities}

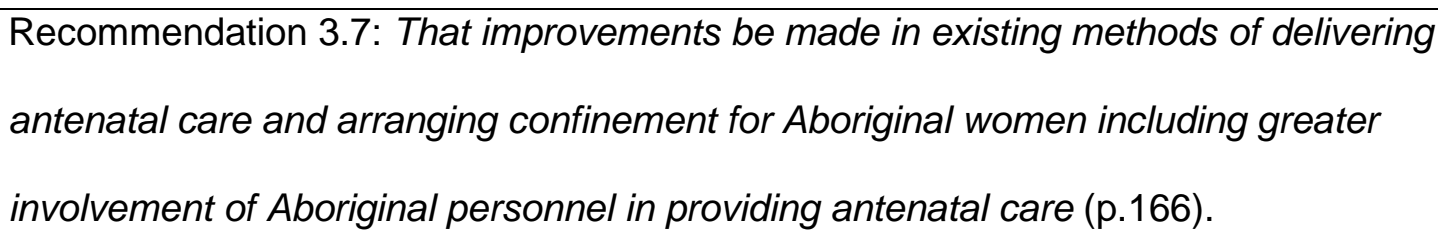

The Shearman Report was one of the first reports in Australia to recognise that Aboriginal and Torres Strait Islander women needed specific maternity services. In 2000, the NSW government established the Aboriginal Maternal and Infant Health Service (AMIHS) to address the maternal and child health needs of Aboriginal and Torres Strait Islander families. One of the government reports that initiated the need for AMIHS was the NSW Aboriginal 
Perinatal Health Report which clearly stated the need for accessible and appropriate maternal health services. ${ }^{10}$ This highlighted the shocking disparities in the health outcomes for Aboriginal families in NSW. There were also higher rates of low birth weight babies, preterm birth and intra-uterine growth restriction. ${ }^{11}$ An evaluation of the AMIHS program in 2005 showed that these targeted support services for Aboriginal and Torres Strait Islander women had positive outcomes. ${ }^{12}$ In particular, an increased proportion of women attended their first antenatal visit before 20 weeks gestation and more women breastfed their babies for longer periods.

Since its inception, AMIHS has developed over 30 targeted antenatal/postnatal programs for Aboriginal women and infants across NSW and put together a comprehensive training and support program for midwives and AHWs. The service also has a number of successful initiatives in place such as the Strong Women Strong Babies Pregnancy Diary, safe sleeping brochures, Building Strong Foundations program, the Quit for a new life smoking cessation program, and the Brighter Futures early intervention program. A further evaluation showed that AMIHS was improving maternity services and outcomes for Aboriginal women, covering around $75 \%$ of Aboriginal births in NSW. ${ }^{13}$ However, the disproportionate difference in morbidity and mortality between Aboriginal and Torres Strait Islander women and babies and other Australian women and babies remains considerable. For example, Aboriginal and Torres Strait Islander women are three times more likely to die in the perinatal period, ${ }^{14}$ and their infants are twice as likely to die compared to other Australian women and their infants. ${ }^{15}$ More recently, the National Maternity Services Plan (2010) reiterated the need to continue improvement of services for Aboriginal women and their families. The focus in the Plan was to enable Aboriginal community-control over services, and ensure cultural competence of hospitals and maternity staff. It also stated that birthing on country programs should be established however these services have not been implemented in many remote areas although they exist in some regional areas in other states (for example, Boodjari Yorgas in 
Armadale, Western Australia, support Nyoongar women to give birth on their country (Whadjuk Boodja).

\section{Visiting rights for privately-practising midwives}

Recommendation 4.18: That the Taskforce recommends that suitably qualified independent midwives be accredited to practise normal midwifery in hospital birth centres and labour wards (p. 200).

Privately-practising midwives (PPM) in Australia care for women during pregnancy, birth and the postnatal period, and often have to, or choose to, do so with little input or formal links with maternity facilities. PPMs are employed by women who wish to give birth at home, who desire a more personal service and do not want to go to hospital. Having 'visiting rights' means that the midwives are accredited to use a particular hospital's facilities, and can mean that, for example, during a homebirth when a woman needs medical intervention, the midwife can transfer her to hospital and continue to be her care provider. Currently in NSW, PPMs do not have visiting rights to public or private hospitals and can only attend women if they are transferred to hospital as a support person or doula.

The Shearman Report recommended that appropriately qualified midwives be accredited to practise normal midwifery in maternity unit's labour wards and birth centres, essentially, have visiting rights. Stipulations were that midwives wishing to have visiting rights needed to formally apply to the hospital and hold professional indemnity insurance, evidence of competence and continuing professional development, willingness to accept hospital policies and participate in quality assurance programs.

By year 2000, the NSW Framework report ${ }^{5}$ reiterated that to expand the range of, and improve the access to options of care in maternity services, there should be formal links developed between PPMs and hospitals that enabled their access to facilities. It went on to recommend the development of protocols for transfer from a planned homebirth to hospital 
facilities, a review of the NSW Homebirth Policy Statement and more integration and collaboration between visiting midwives and formal facilities. At this time, only 5 maternity facilities out of 101 in NSW had accredited visiting midwives ${ }^{5}$ and even this was short-lived due to the collapse of the insurance industry.

\section{Professional indemnity insurance for privately-practising midwives}

In 2001, the Health International Holdings Company (Australia's second largest insurance company) went into liquidation which had a devastating flow-on effect rendering it impossible for PPMs to insure themselves to provide homebirth to women. This was because insurance companies felt that the small number of self-employed midwives premiums were insufficient to cover even a single large award, and so the risk of potential claims was too great.

In 2010, National Registration law required all health practitioners, including midwives, to have professional indemnity insurance in order to practice. ${ }^{16}$ Professional Indemnity Insurance (PII) provides a level of security from civil liability claims, for example, claims for damages, negligence, and also claims of unprofessional conduct. PII for publicly-employed midwives is provided by their health service employer, however, this requirement for PII became a major problem for PPMs providing homebirth. Under this law, PPMs were not only uninsured, but they would be practising unlawfully according to the new National Law. In response to considerable community and professional concerns about the access to homebirth services, the Commonwealth Government provided an exemption for midwives who wished to provide homebirth, and all Australian states amended their National Law to allow this (apart from the Northern Territory). The exemption allowed midwives to provide homebirth legally, but did not solve the problem of insurance; they would still be practising uninsured.

Currently across the country, there are very few PPMs with hospital visiting rights; most of them are in Queensland, with some in other states (Western Australia and Victoria) that have negotiated visiting rights in collaboration with a medical practitioner. ${ }^{17}$ In the National 
Maternity Services Plan, ${ }^{6}$ one of the actions was for the Australian Government to consult with private health insurers through the peak bodies (AHIA and HIRMAA ${ }^{1}$ ) to assist insurers to meet their legislative obligations so that women had more access to primary private midwifery services in the community, as well as in hospital. There is little progress on this issue at present, with no national push (or financial incentive) for hospitals in many states to be obligated to move this issue forward.

\section{Medical intervention in childbirth}

The Shearman report had a focus on normalising birth. As such, it recommended monitoring of the indications for and the rates of medical intervention in childbirth as part of an ongoing interdisciplinary peer review and quality assurance.

Recommendation 4.13: All maternity units should record and monitor the indications for and rates of medical intervention in childbirth as part of ongoing multidisciplinary peer review and quality assurance programs. Where rates for selected procedures consistently exceed the state average, or those of units servicing comparable obstetric populations, a review of maternity unit policy and labour ward procedures should be undertaken (p. 192).

Other recommendations included that women have a birth plan when admitted to the labour ward, outdated practises such as perineal shaving and enemas not be offered and encouragement towards mobilisation in labour. Interestingly, it acknowledged the differing rates of caesarean section for public versus privately insured women, which is something that continues to be the case..$^{18,19}$

Similar to the Shearman report, the NSW Framework report ${ }^{5}$ recognised birth as a normal process and aimed to reduce maternal and perinatal mortality and morbidity through promoting appropriate levels of interventions in maternity care by applying suitable benchmarks for practise. These included reviewing obstetric intervention rates annually,

\footnotetext{
${ }^{1}$ AHIA = Australian Health Insurance Alliance; HIRMAA = Health Insurance Restricted Membership Alliance of Australia
} 
ensuring competency of all providers of maternity care, and developing a 'medication in pregnancy and lactation' service that could be provided by NSW health (MotherSafe) ${ }^{20}$ More recently, the strategy Towards Normal Birth in NSW ${ }^{21}$ focussed solely on reducing intervention, in particular, elective caesarean section, which in 2010 had reached 28.8 per cent of all births in NSW hospitals. ${ }^{22}$

Currently in Australia, birth plans are very common and accepted in practice, feedback on maternity experiences from women is solicited and unnecessary non-evidence based interventions such as perineal shaves and enemas have long since been banished. Most hospitals provide regular case review meetings and morbidity and mortality reviews for all clinicians to attend, and statistical information is freely available regarding a hospital's medical intervention rates. ${ }^{23}$ However, medical intervention in childbirth remains widespread, and rates of caesarean section in particular have nearly doubled in Australia since 1992 (see Table 1). Whilst some women will need medical intervention during labour and birth, it is acknowledged that many have unnecessary procedures that lead to assisted or surgical births without any corresponding lowering of perinatal mortality rates, a higher cost to the tax payer, and possible future health implications for women and babies. ${ }^{18}$

Put TABLE 1 here

Many Local Health Districts have implemented strategies to lower medical intervention rates in childbirth in the form of evidence-based clinical guidelines, vaginal birth after caesarean section (VBAC) clinics and breech clinics (to support selected women having vaginal breech births). International guidelines have also stressed the need for little intervention for low-risk women, for example, abolishing cardiotocographic monitoring upon admission. ${ }^{24}$ Despite the evidence available that promotes normal birth, there are still discrepancies in intervention rates between hospitals, and models of maternity care. In particular, low risk women being 
cared for in a midwifery caseload (continuity of carer) model are $22 \%$ less likely to have a caesarean section, ${ }^{25}$ and women having care in private hospitals are at greater risk of intervention at all levels. ${ }^{18}$

\section{Continuity of maternity care}

The Shearman report recommended that after an evaluation of each community's needs, an appropriate mix of hospital and community based maternity care be implemented. It was stressed that midwifery workforce levels be adequate, and strategies that promote continuity of care be investigated. The British 'Know Your Midwives' scheme was cited as a model to follow. ${ }^{26}$

Recommendation 4.7: Adequate midwifery staffing levels are essential in labour ward and strategies to promote continuity of care should be investigated by maternity units including flexible rostering, cluster or rotation systems and the British 'know your midwife scheme' $\mathrm{p}$. 186.

Shearman suggested that more birth centres become available to all women who were at low or moderate risk of pregnancy/birth complications. Similarly, all maternity units were encouraged to develop home-like non-clinical birth rooms, provide midwives clinics, and foster an attitude that inspired flexible family-centred birth. By 2000, out of the 101 maternity facilities in NSW that offered public maternity services, there were four Birth Centres, 42 midwives clinics, and nine team midwifery programs. ${ }^{5}$ In 2014 , only 10 facilities in NSW have a designated Birth Centre, ${ }^{27}$ although some units may offer rooms that have home-like décor, but the numbers are unknown.

The NSW Framework report in 2000 also emphasised the need to concentrate on implementation of service changes that improved continuity of care and carer. In particular, a coordinated transition from the maternity service to the community and continuity of midwifery care when transferring from birth centre to labour ward, or home to hospital. This 
now occurs in many Australian hospitals, although only a few with publicly-funded homebirth services (15 services Australia-wide) or midwives with visiting rights (mostly in Queensland) remain the lead carer for a woman who is transferred in from a homebirth.

The National Maternity Services Plan (2010) was underpinned by 10 principles for maternity care. One of these was that 'maternity services offer continuity of care across the pregnancy and birthing continuum as a key element of quality maternity care for all women and their babies' (p. 26). Much work has been undertaken to provide this in many institutions, however, many (if not most) women continue to have fragmented care, often from a number of maternity health professionals.

In NSW, we have estimated that of around 50 maternity facilities, approximately $20 \%$ of these offer midwifery-led continuity of care models, mostly to low-risk women and often to small numbers of women attending that hospital. All women need midwifery continuity of care, but women who have higher levels of pregnancy complications needing interdisciplinary care need continuity of care and carer the most. Recent work has shown that women with higher obstetric risks have good outcomes when cared for in this way ${ }^{28}$ and low-risk women have less likelihood of preterm birth and other medical interventions, and are highly satisfied with this type of care. ${ }^{29}$

\section{DISCUSSION}

For the time in which it was written, the Shearman Report was very progressive in its recommendations. In particular, its stance in regard to promotion of midwifery continuity of care, care of Aboriginal women and families, care and resources for non-English speaking women, monitoring of medical intervention, and furthering community-based care were ahead of their time. The approach in the report put women and their babies and families at the centre of care although some of the terminology used (for example, 'confinements', 'deliver') are indicative of the time. Our paper focusses on only five of the Shearman 
Report's 105 recommendations but these are the 'big picture' issues common to many other national and NSW maternity reports conducted over the last quarter of a century.

Since the Shearman Report, maternity services in NSW have addressed many issues relating to increased options of maternity models of care for women, including support programs that allow women to go home early from hospital, introduced lactation consultants to ensure consistency of breastfeeding advice/care, and peer review systems to ensure quality of care. The Report had extensive recommendations for improvements in care for vulnerable women, including those with mental illness, drug and alcohol dependency, low socioeconomic groups, and also those with stillborn or sick infants.

One of NSW Health's achievements since the Shearman Report recommendations has been the successful implementation of the Aboriginal Maternal and Infant Health Strategy (AMIHS). This strategy, put in place to address the disparity between maternal and neonatal outcomes of Aboriginal and non-Aboriginal women and babies, is now an integral part of maternity care for this group of women throughout the state. Since the introduction of AMIHS, the infant mortality rate has decreased by $60 \%$ to 3.8 deaths per 1000 live births in the period 2010-2012 (from 9.5 deaths in 2000-2002) ${ }^{30}$ This is a significant closing of the gap in infant mortality rates. The AMIHS strategies, based on primary health care principles and partnership with Aboriginal peoples, has shown marked improvements in access to care and outcomes over the last 15 years. ${ }^{13}$

Unfortunately, more than 25 years after the Shearman Report, many recommendations for improving maternity care have not been carried out, or have only half-heartedly been put in place. For example, allowing hospital visiting rights for privately-practising midwives and offering women midwifery continuity of care during their pregnancies, birth and postnatal periods are not universally available across the state.

There are very few privately-practising midwives due to a lack of Professional Indemnity Insurance, and those that do work in this way often do not have hospital visiting rights, and 
can even face hostility from other health professionals when transferring women to hospital. ${ }^{31}$ Australia-wide publicly-funded homebirth services appear to work well with favourable outcomes, ${ }^{32}$ but again, these are not universally available.

There has been some provision of continuity of care models in maternity services in Australia. However, only a small proportion of women are cared for in this way and most women still experience fragmented care through a hospital antenatal clinic without a relationship with a known midwife. Every maternity service report since 1989 has stressed the importance of improving continuity of care, and in particular, establishing midwifery-led continuity of care models. This type of care has high level evidence to support it and evidence of good maternal and neonatal outcomes, maternal satisfaction, cost-effectiveness and efficacy for women at all levels of obstetric risk. ${ }^{25,28,29}$ Considering these factors, all women should be offered midwife-led continuity of care during their pregnancies, and these services should be developed as a matter of urgency.

The politics of maternity services in Australia remains dominated by a strong medical sector and a financial imperative to retain the status quo. Public health funding is often concentrated on the acute care hospital sector, large subsidies continue to be paid to the private sector by the federal government, there are taxation incentives to buy private health insurance thus diverting funds and attention from the public sector and multiple restructures of local health districts cause staff upheaval. ${ }^{3}$

There is an urgent need to garner political will at both state and national level as well as high $\underline{\text { level support being required from hospital and health system management, professional }}$ $\underline{\text { associations and the consumer movement. Nursing unit managers in hospitals need support }}$ to understand the flexible ways in which midwives want to work, and can safely work, to provide midwifery continuity of care. Recognising the unique role of the midwife and ensuring care is always focussed on the woman and her baby is fundamental to widespread reform. $^{33}$ 
The National Maternity Services Plan ${ }^{6}$ comes to an end in 2015 and there is still a considerable amount of to do - some incomplete actions, as we have shown, date back to The Shearman Report and are as relevant today as they were 25 years ago. Midwifery continuity of care, as well as other innovative recommendations from the Shearman Report have not been implemented extensively in Australia, hence there is a clear need for continued advocacy to bring about change in maternity services. Government reports are valuable in instigating change, but there needs to be further development and creativity in how to ensure facilities develop these important services.

\section{CONCLUSION}

The Shearman Report did have some impact on the obstetric-dominated maternity services in NSW, however, impediments to ensuring services become, and remain, woman-centred and community-based remain strong. The Shearman report was a comprehensive, forwardthinking report that covered a broad range of issues including innovative care of women throughout the spectrum of pregnancy, birth and the postnatal period, planning of obstetric beds, parenting education, rural maternity services, and care of women from vulnerable groups and communities. This paper outlines the progress (or lack of progress) made on five key recommendations: community-based maternity care, rural maternity services, visiting rights for privately-practising midwives, medical intervention in childbirth and continuity of midwifery led care. Despite implementation of services and innovation in some of these areas, recommendations have not been carried out in all maternity facilities, especially rural units, and the issue of visiting rights for privately-practising midwives has taken a backwards step since 1989. 


\section{REFERENCES}

1. NSW Health. Maternity Services in NSW: final report of the Ministerial Task Force on Obstetric Services in NSW. Sydney: NSW Department of Health, 1989.

2. Schofield T. Politics, Public Policy and Childbirth, NSW 1950-1990: University of Sydney; 1995.

3. Reiger K. A neoliberal quickstep: contradictions in Australian maternity policy. Health Sociology Review 2006; 15(4): 330-40.

4. Reiger K. Birthing in the postmodern moment: Struggles over defining maternity care needs. Australian Feminist Studies 1999; 14(30): 387-404.

5. NSW Health. The NSW Framework for Maternity Services. Sydney: NSW Health Department, 2000.

6. Commonwealth of Australia. National Maternity Services Plan. Canberra: Department of Health, 2010.

7. Homer CS, Davis GK, Brodie PM, et al. Collaboration in maternity care: a randomised controlled trial comparing community-based continuity of care with standard hospital care. BJOG: An International Journal of Obstetrics \& Gynaecology 2001; 108(1): 1622.

8. Commonwealth of Australia. Maternal and Perinatal Committee Report on Obstetric Services; Part II Country Regions. Canberra: Australian government, 1983.

9. Rural Doctors Association of NSW. RDAA response to Improving Maternity Services in Australia: A discussion paper from the Australian Government. no date. http://www.rdaa.com.au/Uploads/Documents/RDAA\%20Submission\%20to\%20the\%20Mater nity\%20Services\%20Review_20101015110159.pdf (accessed June 1 2015).

10. NSW Department of Health. The Aboriginal Perinatal Health Report. Sydney: NSW Department of Health, 2003.

11. NSW Department of Health. New South Wales mothers and babies 1999: NSW Department of Health, 2001.

12. NSW Health. NSW Aboriginal Maternal and Infant Health Strategy evaluation: final report 2005. Sydney: NSW Health, 2005.

13. Murphy E, Best E. The Aboriginal Maternal and Infant Health Service: a decade of achievement in the health of women and babies in NSW. New South Wales Public Health Bulletin 2012; 23(3-4): 68-72.

14. Johnson S, Bonello M, Li Z, Hilder L, Sullivan E. Maternal deaths in Australia 20062010, Maternal deaths series no. 4. Cat. no. PER 61. Canberra: AlHW, 2014. 
15. Australian Institute of Health and Welfare, Australian Bureau of Statistics. The Health and Welfare of Australia's Aboriginal and Torres Strait Islander Peoples. Canberra: ABS, 2012.

16. ACT Government. Health Practitioner Regulation National Law Canberra: ACT Governement; 2010.

17. MyMidwives. Personalised Pregnancy, Birth and Post Birth Care for all women and their families. 2015. http://mymidwives.com.au/ (accessed 10 May 2015).

18. Dahlen HG, Tracy S, Tracy M, Bisits A, Brown C, Thornton C. Rates of obstetric intervention among low-risk women giving birth in private and public hospitals in NSW: a population-based descriptive study. BMJ Open 2012; 2(5).

19. Roberts C, Algert C, Douglas I, al. e. Trends in labour and birth interventions among low-risk women in New South Wales. Aust N Z J Obstet Gynaecol 2002; (42): 176-81.

20. South Eastern Sydney Local Health District. MotherSafe. 2015.

http://www.mothersafe.org.au/ (accessed May 10 2015).

21. NSW Kids and Families. Maternity - Towards Normal Birth in NSW. Sydney: Ministry of Health NSW, 2010.

22. Centre for Epidemiology and Research. New South Wales Mothers and Babies, 2006. Sydney: NSW Department of Health, 2007.

23. Centre for Epidemiology and Evidence New South Wales. Mothers and Babies 2012. Sydney: NSW Ministry of Health, 2014.

24. National Institute for Health and Care Excellence. Intrapartum care: care of healthy women and their babies during childbirth. NICE clinical guideline 190. London: National Collaborating Centre for Women's and Children's Health; 2014.

25. McLachlan H, Forster D, Davey M-A, et al. Effects of continuity of care by a primary midwife (caseload midwifery) on caesarean section rates in women of low obstetric risk: the COSMOS randomised controlled trial. BJOG: An International Journal of Obstetrics \& Gynaecology 2012; 119(12): 1483-92.

26. Flint C, Poulengeris P, Grant A. The 'Know Your Midwife' scheme-a randomised trial of continuity of care by a team of midwives. Midwifery; 5(1): 11-6.

27. Pregnancy Birth and Beyond. List of Australian Birth Centres. 2015.

http://www.pregnancy.com.au/birth-choices/birth-centres/australian_birth_centres.shtml (accessed 13 May 2015).

28. Tracy SK, Hartz DL, Tracy MB, et al. Caseload midwifery care versus standard maternity care for women of any risk: M@ NGO, a randomised controlled trial. The Lancet 2013; 382(9906): 1723-32.

29. Sandall J, Soltani H, Gates S, Shennan A, Devane D. Midwife-led continuity models versus other models of care for childbearing women. The Cochrane Library 2013. 
30. Australian Bureau of Statistics. Deaths. Catalogue number 3302.0. Canberra: Centre for Epidemiology and Evidence, NSW Ministry of Health, 2012.

31. Fox D, Sheehan A, Homer C. Experiences of Women Planning a Home Birth Who Require Intrapartum Transfer to Hospital: A Metasynthesis of the Qualitative Literature. International Journal of Childbirth 2014; 4(2): 103-19.

32. Catling-Paull C, Coddington R, Foureur M, Homer C. Publicly funded homebirth in Australia: a review of maternal and neonatal outcomes over 6 years. Medical Journal of Australia 2013; 198(11): 616-20.

33. Renfrew MJ, McFadden $\mathrm{A}$, Bastos $\mathrm{MH}$, et al. Midwifery and quality care: findings from a new evidence-informed framework for maternal and newborn care. The Lancet 2014; 384(9948): 1129-45. 